\title{
Improving Teamwork and Communication through the Use of TeamSTEPPS
}

\author{
Amanda Curtsinger \\ Capella University, Minneapolis, MN, USA \\ Email: amanda.curtsinger@yahoo.com
}

How to cite this paper: Curtsinger, A. (2018) Improving Teamwork and Communication through the Use of TeamSTEPPS. Open Access Library Journal, 5: e4816. https://doi.org/10.4236/oalib.1104816

Received: July 31, 2018

Accepted: August 25, 2018

Published: August 28, 2018

Copyright ( $\odot 2018$ by author and Open Access Library Inc.

This work is licensed under the Creative Commons Attribution International License (CC BY 4.0).

http://creativecommons.org/licenses/by/4.0/

\begin{abstract}
Effective teamwork and communication are critical components for quality and patient safety. Investing in the nursing leadership team strengthens the collaboration between all health care professionals. TeamSTEPPS is an evidence based program that has been shown to improve the quality and safety by fostering effective teamwork and communication skills. In this quality improvement project, TeamSTEPPS 2.0 training was used to improve leadership skills among nurses in leadership roles on a mid-sized medical surgical unit. Nursing leadership was evaluated using the TeamSTEPPS Learning Benchmarks Quiz, the TeamSTEPPS Teamwork Perceptions Questionnaire (T-TPQ), and the TeamSTEPPS Observation Tool. The results revealed an improvement in teamwork and communication among the staff and nursing leadership over a ten-week period.
\end{abstract}

\section{Subject Areas}

Nursing

\section{Keywords}

TeamSTEPPS, Communication, Teamwork, Leadership

\section{Introduction}

On a medical surgical unit in a small Southern State suburban hospital, it was determined that the lack of formal leadership training was leading to poor communication and teamwork. This has led to a low percentage of staff indicating that they feel trust in their nursing leaders at only 78\%, according to the 2016 employee engagement survey. Considering that professional development for nurse leaders is essential to support a strong nursing care environment, an intervention was necessary. The goal of this project was to implement a sustainable 
nurse leadership training program in order to increase organizational support and team cohesion [1].

\section{Available Knowledge}

Through an exhaustive review of literature, a number of studies were reviewed. CINAHL, Cochrane Library, Medline, and EBSCO were the primary databases that were searched; the American Nurses Association (ANA), The American Association of Critical Care Nurses (AACCN), and Press Ganey were also valuable websites for this project. By typing in nurse leader, 18,187 articles were produced, continuing professional development had 9717 articles, and continuing education came up with 66,014 . The search was refined to the English language in only peer reviewed journals within the last five years. Editorials were excluded. Additional keywords included professional practice environment, nurses, leaders, managers, hospital, job satisfaction, employee retention, quality of care, nurse empowerment, leadership training.

Of the 85 studies retrieved, 32 studies were retained for further review. Of the 32, 26 studies were found to be fitting for this specific project. Most studies focused on characteristics that contribute to a professional nursing environment and discussed factors related to job satisfaction, nurse retention, nurse burnout, and quality of care. Studies chosen included quantitative, qualitative, and systematic reviews of the literature. The selected peer-review journal articles ranged in time from 2008 to 2017, with the majority of the articles written in the past five years. By using the levels of evidence provided by Melnyk and Fineout-Overholt, studies were graded for evidence, using a range of Level 1 through Level 4 [2]. The levels of evidence are assigned to the studies based on the quality and validity ranging a systematic review (level I) to evidence from a case-controlled study (level IV). The levels are indicative of how strong of a recommendation the study is to a particular project. The majority of studies evaluated for this project fell between level II and III.

\section{Project Design}

The TeamSTEPPS 2.0 was a quality improvement initiative that used the TeamSTEPPS program to increase communication and teamwork in a mid-sized hospital. The project was conducted prospectively and included the collection quantitative data including using a pre/post survey. The PDCA method was used to test the effectiveness of the evidence based training program [3]. The PDCA model is outlined as follows:

- Plan-The pre-intervention phase included reviewing previous literature and performing a needs assessment at the site. Planning also included an assessment of available resources.

- Do-The implementation phase included leadership training using the educational program and baseline evaluation of team concepts.

- Check-Included an assessment of leadership competency data collection and analysis. 
- Act to hold the gain-An assessment plan for continuous improvement and plan for incorporating TeamSTEPPS into on-going leadership training will be developed.

\subsection{Setting}

The study was conducted at a mid-sized community hospital on a medical surgical unit. The unit employs approximately 80 staff nurses and 60 personal care assistants. The unit holds 48 beds, which are consistently full. Ten staff nurses, four aides, and one unit secretary staff are on the unit on a regular day. Each nurse has a maximum of five patients at a time, and each aide has a maximum of 12.

\subsection{Population and Sample of Nurse Leaders}

The sample for this project consisted of nurse leaders on the medical surgical unit who represent a larger population of leaders at this organization. Of the eight dayshift nurse leaders on the unit, seven are female. The ages of nurse leaders range from 24 to 36 , and the tenure at the hospital ranges between three to 12 years. The characteristics of the nurse leaders on this unit are consistent with the nurse leaders of other units. As an organization, only an associate's degree and minimal tenure at the hospital are required for a leadership position.

\subsection{Inclusion and Exclusion Criteria}

All dayshift nurse leaders from the medical surgical unit were encouraged to participate in the TeamSTEPPS program. Those who were excluded were the staff nurses who do not hold a leadership position. Also, other floors were not asked to participate at this time. All nurse leaders were asked to participate in taking the TeamSTEPPS Learning Benchmark quiz before and after accessing the TeamSTEPPS 2.0 program. Staff nurses, personal care assistants (PCA's), medical doctors, unit secretaries, and other professionals on the unit participated in the TeamSTEPPS Teamwork Perceptions Questionnaire (T-TPQ) at the beginning and end of the intervention period. Those excluded from taking the questionnaire were any staff from different units other than the medical surgical unit.

\section{Intervention}

Three tools were used to assess the impact TeamSTEPPS had on the unit. The TeamSTEPPS Learning Benchmark Quiz, the TeamSTEPPS Observation Tool, and the TeamSTEPPS Team Perceptions Questionnaire were all developed to be used with the TeamSTEPPS program. All three have been established as reliable measurement tools by the developers of the program. The quizzes and questionnaires were administered by the project manager, and each observation tool was filled out by the project manager to increase the accuracy in recorded observations. 


\subsection{TeamSTEPPS Learning Benchmark Quiz}

Before the TeamSTEPPS 2.0 program was taken, each nurse leader was asked to fill out the TeamSTEPPS Learning Benchmarks quiz. The TeamSTEPPS Learning Benchmark Quiz includes 23 multiple choice questions. Each correct answer is worth approximately 4.3 points on a 100 point scale. Tests were scored and reported as the percentage correct. To protect the identity of the subjects, results were tallied and a mean score for the group was determined. The pretest and posttest were both conducted with this method. Participation in the TeamSTEPPS training and quizzes initiative was voluntary and participation was deemed as consent.

\subsection{TeamSTEPPS Team Perceptions Questionnaire}

The T-TPQ results were recorded in an excel program. Consisting of 35 questions, this questionnaire uses a five point Likert Scale in which participants score each question from strongly disagree to strongly agree according to their perception of leadership [4]. The T-TPQ test has demonstrated an alpha between .83 and 0.94 [5]. The five domain measurements in the TeamSTEPPS program include team structure, leadership, situation monitoring, mutual support and communication. The five domains are discussed in great detail in the TeamSTEPPS 2.0 program, and a change in the scores reflects the impact the program had on the nurse leaders.

\subsection{TeamSTEPPS Observation Tool}

The TeamSTEPPS Observation Tool was used to determine if the nurse leaders were able to apply the information learned from the program to every day practice. The project manager used this tool to assess huddles, at random, once per week. The learner was able to rate the performance of the nurse leaders on a five-point Likert Scale of one to five, which is very poor to excellent respectively. Chao Zhang and associates demonstrated test-retest reliability of 0.707 [6]. One unintended result of the huddle routine was how serious the leaders took the initiative to optimize the huddle. After reviewing the importance of huddles during the TeamSTEPPS training, it was mandatory for staff to attend them. A roster is now filled out at every huddle to ensure the huddle is conducted and that all staff is present.

\section{Ethical Considerations and Project Approval}

In order to protect the identity of each participant, there were no identifying markers on any quizzes or questionnaires. Additionally, the staff did have the right to take these surveys in private so that no one else could see their answers. Each form was then placed in a manila envelope. This protected the staffs privacy rights and helped the staff answer the questions more honestly. IRB approval from the hospital was sought and granted to ensure human subject protection given the sensitivity of the information. During this project, there was no 
patient interaction and therefore no ethical considerations in regards to patient protected health information.

\section{Analysis}

There were a total of seven nurse leaders who participated in the TeamSTEPPS 2.0 training. Therefore, seven TeamSTEPPS Learning Benchmarks pre and posttests were taken. The scores from the pre and posttest were aggregated and displayed as an overall group score. Interval data produced from the two tests was then compared to determine if the TeamSTEPPS 2.0 program increased knowledge regarding teamwork and communication.

The T-TPQ surveys were voluntary and anonymous. A total of 27 pre-surveys were completed, as well as 25 post surveys. The questionnaire uses a five-point Likert scale, in which the ordinal data rages from one (strongly agree) to five (strongly disagree). Each of the 35 questions was analyzed looking at the overall variance of the means of the pre and post results. The 35 questionnaire survey was not altered in any way to maintain its validity, and no descriptive statistics were added.

The Team Performance Observation Tool also measured differences in survey scores over time. The Observation Tool was used at random once a week for six weeks straight. Data from the Observation Tool was compared over time, from baseline to the end of the project to determine overall leadership competency in teamwork and communication demonstrated during MDI huddles.

\section{Results}

\subsection{T-TPQ}

A two sample $t$-test was used to measure each of the survey questions, as well as the aggregated scores in each of the five categories with a 95\% confidence level. Over half of the questions had a significant improvement in mean score between the pre and post surveys. Only one question displayed a decrease in mean score between the pre and post survey. There was a $0.02 \%$ decrease in score when asked if staff allow the patients plenty of time to ask questions during communication. The lowest gains were also noted in questions that regarded patient advocacy, yet there was a high gain in conflict resolution from the management.

Additionally, the results derived from the five sub-groups showed significant improvement. The categories of leadership and situation monitoring had the greatest improvements, while communication and team function showed the least. However, considering that the $p$-value of all five categories are lower than 0.05 , we are able to conclude that the average score of the post survey scores are significantly higher than the scores of the pre survey.

\subsection{Learning Benchmark Quiz}

The Learning Benchmark Quiz tested the nurse leaders' knowledge on how to handle certain situations. A total of seven nurse leaders took the quiz, which 
consisted of 23 questions. Many of the questions were common knowledge; hence the high scores from the pre-test. However, there was content discussed through the TeamSTEPPS 2.0 program that the leaders were able to learn from. Because the scores were anonymous, they needed to be aggregated and compared to the pre and posttest. On the first test, the average points missed was 3.43 , while the average missed on the posttest was 1.43 . With a student's $t$-test of 0.002 , it would conclude that the average score of the posttest is significantly larger than the average.

\subsection{TeamSTEPPS Observation Tool}

Throughout the six weeks, the leaders' behaviors on the unit were monitored. By using the TeamSTEPPS Observation Tool, the five core components were rated by the learner. On the first day of training, the tool was filled out to determine a baseline. At the time, it was not mandatory to have all staff attend the huddles. Also, without the tools gained with the MDI Huddle Board, the huddles were unorganized and did not cover all topics. Through the TeamSTEPPS 2.0 training and the tools gained by the MDI Huddle board, the behaviors on the unit began to shift. Huddles became mandatory and much more organized. Staff was also encouraged to participate in the huddles, which increased morale and gave them a sense of inclusion during the shift.

The Observation Tool also showed an improvement in the nurse leaders' behaviors over time. The tool used a five point Likert scale, ranging from 1 (very poor) to 5 (excellent). Keeping in mind that the leaders were aware of the observer's presence, they may have given more effort to apply their skills during those particular shifts. Regardless, according to the results there was a significant improvement in the team function on the unit, implying that teamwork did increase over the six weeks.

\section{Summary}

The TeamSTEPPS program was designed to improve safety, quality, and efficiency in healthcare [7]. The goal of this project was to give the nurse leaders the necessary skills to be able to effectively lead a team, resolve conflicts, and improve performance. By implementing the TeamSTEPPS strategies, a reduction in errors and an increase in patient care will ensue. Therefore, many positive outcomes are as a result of investing in the nursing leadership by implementing the TeamSTEPPS training.

As shown by the results from the Learning Benchmark Quiz, the TeamSTEPPS Observation Tool, and the T-TPQ, the program did demonstrate to be effective. The team leaders have learned new skills from the program and are able to effectively apply them to every day practice. Staff members have also expressed their positive opinions of the change in the atmosphere of the unit, especially with the increase in communication and teamwork. The anecdotal increase in staff satisfaction due to the improvements in the work environment adds to the strength of the project. 


\section{Conflicts of Interest}

The author declares no conflicts of interest regarding the publication of this paper.

\section{References}

[1] Collini, S., Guidroz, A. and Perez, L. (2015) Turnover in Health Care: The Mediating Effects of Employee Engagement. Journal of Nursing Management, 23, 169-178. https://doi.org/10.1111/jonm.12109

[2] Melnyk, B. and Fineout-Overholt, E. (2010) Evidence-Based Practice: Step by Step. The Seven Steps of Evidence-Based Practice: Following This Progressive, Sequential Approach Will Lead to Improved Healthcare and Patient Outcomes. American Journal of Nursing, 110, 51-53. https://doi.org/10.1097/01.NAJ.0000366056.06605.d2

[3] Peer, K. and Rakich, J. (2000) Accreditation and Continuous Quality Improvement in Athletic Training Education. Journal of Athletic Training, 35, 188-193.

[4] Castner, J. (2012) Validity and Reliability of the Brief TeamSTEPPS Teamwork Perceptions Questionnaire. Journal of Nursing Measurement, 20, 186-198.

https://doi.org/10.1891/1061-3749.20.3.186

[5] TeamStepps (2017) Agency for Healthcare Research and Quality. https://www.ahrq.gov/teamstepps/index.html

[6] Zhang, C., Miller, C., Volkman, K., Meza, J. and Jones, K. (2014) Evaluation of the Team Performance Observation Tool with Targeted Behavioral Markers in Simulation-Based Interprofessional Education. Journal of Interprofessional Care, 29, 202-208. https://doi.org/10.3109/13561820.2014.982789

[7] King, H., Battles, J., Baker, D., Alonso, A., Salas, E., Webster, J., Toomey, L. and Salisbury, M. (2008) TeamSTEPPS: Team Strategies and Tools to Enhance Performance and Patient Safety. Agency for Healthcare Research and Quality, 15, 1-2. 